\title{
summary
}

\section{Occlusal splints may be of benefit in $T M D$, but there is little evidence for the use of occlusal adjustment}

Forssell H, Kalso E, Koskela $P$, Vehmanen $R$, Puukka $P$, Alanen $P$. Occlusal treatments in temporomandibular disorders: a qualitative systematic review of randomized controlled trials. Pain 1999; 83:549-561

Objective A systematic review of occlusal splints and occlusal adjustments in the treatment of temporomandibular disorders (TMD).

Data sources Medline (1966-1999), Embase, Index Medicus (19661980), Cochrane Controlled Trial Register, DARE and reference lists of retrieved papers. There were no language exclusions.

Study selection Studies were included if they were randomised comparisons of occlusal splint therapy or occlusal adjustments to treat TMD and placebo, no-treatment or other interventions. Studies were scored for quality.

Results Eighteen studies met the inclusion criteria, 14 of splint therapy and four of occlusal adjustment. The overall quality of the trials was fairly low: the mean quality score was 0.43 (range, $0.12-0.78$ ) out of a maximum score of 1.00 . The most obvious methodological shortcomings were inadequate blinding, small sample sizes, short follow-up times, great diversity of outcome measures and numerous control treatments, some of unknown effectiveness. Splint therapy was found to be superior to three and comparable to 12 control treatments, and superior or comparable to four passive controls. Occlusal adjustment was found to be comparable to two and inferior to one control treatment, and comparable to the passive control in one study.

Conclusion Occlusal splints may be of some benefit in the treatment of TMD but evidence for the use of occlusal adjustment is lacking. There is obviously a need for well-designed controlled studies to analyse current clinical practices.

Address for reprints: Heli Forssell, Department of Oral Diseases, Turku University Central Hospital, Lemminkaisenkatu 2, FIN-20520 Turku, Finland. Fax +358 23338248. E-mail: heli.forssell@tyks.fi

\section{Commentary}

This excellent assessment of randomised clinical trials (RCT) gives a clear takehome message on occlusal studies. The authors elucidate important considerations in the quality assessment of RCT, including subject compliance, dropout and the calibration of examiners. Another critical limitation in clinical trials that include patients who have orofacial pain is the use of broad inclusion and exclusion criteria for selection of subjects. This results in the "it makes no difference what you do" findings of most clinical trials on TMD patients. Strict adherence to selection criteria limiting subjects to one diagnostic group should lead to applicable evidence upon which to base future treatments for patients who have that diagnosis.

The review's comparison of the highquality splint studies by Dao et $a l^{1}$ and Ekberg et $a l^{2}{ }^{2}$ which were of identical design but different results, illustrates this point. Although the latter study found a benefit by measuring the subject's perceived pain relief, the former did not use this outcome measure. Because the sub- jects of Ekberg et al were arthralgia patients whereas those of Dao et al had myofascial pain, one might expect a different result. In addition, although both tested stabilisation splints, Dao's subjects wore theirs for 24 hours, compared with only night-time wear for those in the Ekberg et al study. No wonder the outcomes in these two studies were contradictory; they examined subjects with different diagnoses with different treatments, by different outcome measures.

Not only do differences in subject selection and treatment make it difficult for a clinician to compare RCTs with their current patient care, they also prevent the duplication of results in subsequent studies. The replication of positive findings is critical before a treatment, especially a non-reversible one, should be considered. But, of course, there are exceptions.

In conclusion, there are two take home messages.

1. The clinician's perceived benefit of occlusal splint therapy is only modestly supported in the literature, while lack of support for occlusal adjustment suggests it may be con- tra-indicated for the treatment of temporomandibular disorders. In other words the benefit from splint therapy doesn't indicate the need for occlusal rehabilitation ${ }^{3}$.

2. Only when a patient becomes dependent upon full time splint wear for symptom relief, and taking the appliance away results in return of symptoms, should one consider irreversible occlusal treatments

1. Dao TTT, Lavigne GJ, Charbonneau A, Feine JS, Lund JP. The efficacy of oral splints in the treatment of myofascial pain of the jaw muscles: a controlled clinical trial. Pain 1994; 56:85-94.

2. Ekberg EC, Vallon D, Nilner M. Occlusal appliance therapy in patients with temporomandibular disorders. A double-blind controlled study in a short-term perspective. Acta Odontol Scand 1998; 56:122-128.

3. Clark GT. A critical evaluation of orthopedic interocclusal appliance therapy: design, theory, and overall effectiveness. J Am Dent Assoc 1984; 108:359-364.

Jeffry R Shaefer Harvard School of Dental Medicine, Boston, Massachusetts, USA 